\title{
Metabolic tumor volume on PET reduced more than gross tumor volume on CT during radiotherapy in patients with non-small cell lung cancer treated with 3DCRT or SBRT
}

\author{
Pawinee Mahasittiwat • Shuanghu Yuan • \\ Congying Xie • Timothy Ritter • Yue Cao • \\ Randall K. Ten Haken • Feng-Ming Spring Kong
}

Received: 16 January 2013 / Accepted: 21 January 2013 /Published online: 26 April 2013

(C) Springer-Verlag Berlin Heidelberg 2013

\begin{abstract}
Objective We have previously demonstrated that tumor reduces in activity and size during the course of radiotherapy (RT) in a limited number of patients with non-small cell lung cancer (NSCLC). This study aimed to quantify the metabolic tumor volume (MTV) on positron emission tomography (PET) and compare its changes with those of gross tumor volume (GTV) on computed tomography (CT) during-RT for 3D conformal radiotherapy (3DCRT) and stereotactic body radiotherapy (SBRT).

Methods Patients with stage I-III NSCLC treated with a definitive course of RT \pm chemotherapy were eligible for this prospective study. FDG-PET/CT scans were acquired within 2 weeks before RT (pre-RT) and at about two thirds of total dose during-RT. PET metabolic tumor volumes (PET-MTVs) were delineated using a method combining the tumor/aorta ratio autosegmentation and CT anatomybased manual editing. Data are presented as mean $(95 \%$ confident interval).

Results The MTV delineation methodology was first confirmed to be highly reproducible by comparing volumes
\end{abstract}

P. Mahasittiwat $\cdot$ S. Yuan $\cdot$ C. Xie $\cdot$ T. Ritter $\cdot$ Y. Cao $\cdot$

R. K. Ten Haken · F.-M. S. Kong $(\bowtie)$

Department of Radiation Oncology, University of Michigan

Medical Center, Box 0010,

1500 E. Medical Center Dr,

Ann Arbor, MI 48109, USA

e-mail: fengkong@med.umich.edu

P. Mahasittiwat

Division of Radiation Oncology, Department of Radiology, Faculty of Medicine Siriraj Hospital, Mahidol University,

Bangkok, Thailand

T. Ritter

Department of Radiation Oncology, Veteran Administration

Health Center, Ann Arbor, MI, USA defined by different physicians and using different systems (coefficiency $>0.98$ ). Fifty patients with 88 primary and nodal lesions were evaluated. The mean ratios of MTV/GTV were $0.70(-0.07 \sim 1.47)$ and $0.33(-0.30 \sim 0.95)$ for pre-RT and during-RT, respectively. PET-MTV reduced by $70 \%(62-$ $77 \%)$, while CT-GTV by $41 \%(33-49 \%)(p<0.001)$ during-RT. MTV reduction was $72.9 \%$ and $15.4 \%$ for 3DCRT and SBRT, respectively $(p<0.001)$.

Conclusion PET-MTV reduced more than CT-GTV duringRT, while patients treated with 3DCRT reduced more than SBRT. RTOG1106 is using during-RT PET-MTV to adapt radiation therapy in 3DCRT.

Keywords Metabolic tumor volume · GTV · During radiotherapy $\cdot$ Non-small cell lung cancer

\section{Introduction}

$\left[{ }^{18} \mathrm{~F}\right]$ Fluorodeoxyglucose (FDG) positron emission tomography (PET) for tumor metabolic activity has been widely used in patients with non-small cell lung cancer (NSCLC) in diagnosis, staging, restaging, treatment response assessment, and radiation therapy planning. FDG-PET plays an important role in target delineation in radiation treatment planning for NSCLC [1-6]. Use of FDG-PET improves the accuracy of target definition [3, 7]. For primary tumors, FDG-PET helps differentiating tumor from collapsed lung, adjacent normal tissue such as large vessels, and defining disease extent in chest wall. PET scans reduce inter-observer variability compared with computed tomography (CT) alone. Integrated PET-CT scans further improve delineation consistency $[8,9]$. While most studies have focused on preRT imaging for RT planning, or post-treatment PET for 
treatment response assessment, it is largely unknown whether changes occur during radiotherapy, which, if they are possible to assess, may provide an opportunity to redirect the remaining treatment.

We have previously demonstrated in a small pilot study that tumors reduce in activity during-RT [10], and duringRT, metabolic tumor volume (PET-MTV) can be used to adapt radiation treatment to provide radiation dose escalation (30-102 Gy; mean, $58 \mathrm{~Gy}$ ) to more active malignancies or to reduce normal tissue complication probability by 0.4 $3 \%$ (mean, $2 \%$ ) on dry run dosimetry studies [11]. However, it is challenging to define MTV consistently because tumor margins are indistinct, due to heterogeneous [18] FDG uptake distribution and limited spatial resolution. The best target delineation criteria have not yet been established [12]. Currently, PET scanning is often used only to define the location of tumor, and if MTV is defined, methods used for definition vary among investigators in the literatures. In general, there are two basic strategies: (1) manual delineation based on visual inspection, depending on human skill and judgment, (2) using automated or semi-automated computer algorithms to identify the tumor boundary, which may be based on a fixed standard uptake value (SUV), a threshold of tumor maximum, or a fixed tumor to background ratio [13]. For tumor volume-based adaptive RT, one should also note that gross tumor volume on CT (CT-GTV) also reduces during the course of RT $[14,15]$. It is unknown whether there is any difference between changes of PET metabolic tumor volumes (PET-MTV) and CT-GTV.

We hypothesized that PET-MTV can be delineated relatively objectively by a method combining strengths of above two strategies and that there is greater reduction of MTV than GTV during-RT. We tested hypotheses through the following ways: (1) study the reproducibility of the proposed method, (2) define PET-MTV and CT-GTV pre- and during-RT, and (3) study the changes and correlations between MTV and GTV during-RT. Additionally, with wide availability of stereotactic body radiation (SBRT), we investigated the changes of tumor volume changes after a few fractions of hypofractionated SBRT on PET and CT and compared differences in volumetric changes between 3DCRT and SBRT.

\section{Methods}

Study population

Eligible subjects included those with stage I to III NSCLC enrolled in IRB-approved prospective lung treatment and imaging protocols. All patients received a definitive course of conformal RT with or without chemotherapy and had a
PET-CT before and during the course of treatment. Patients with stage I or II disease underwent daily fractionated (2.0 to 3.4 Gy fraction size) radiotherapy or hypofractionated SBRT (10-20 Gy fraction size); patients with stage III disease were treated with concurrent and adjuvant carboplatin/paclitaxel under a prospective clinical trial, in which patients may receive higher doses than in common practice. The dose of RT for the treatment protocol patients was based on an estimated normal lung complication probability of 15-17\%. Patients with prior thoracic RT were excluded from the study.

Study design

The FDG-PET/CT scans were acquired within 2 weeks before RT (pre-RT) and during the course of radiation therapy (during-RT) after the delivery of approximately 45 in 2 Gy equivalent of 3DCRT, as described previously [10], or $2 / 3$ prescription SBRT. The reason for selecting this time point for during-RT PET scan is to make future adaptive therapy possible if these volumes are meaningful. The FDGPET/CT scan was performed in a standard fashion on a flat table top. The PET images were obtained beginning approximately $60 \mathrm{~min}$ after administration of 8 to $10 \mathrm{mCi}$ of ${ }^{18}$ FDG. The CT images (5-mm slices) for the PET/CT study were acquired during quiet breathing. Contrast-enhanced CT scans were also acquired in standard treatment position, at the end of inhale, exhale, and free-breathing states.

Tumor volume delineation: general principles

PET-CT images from the diagnostic radiology department were transferred to the functional image analysis tool (FIAT) and the UM-Plan system (in-house planning systems). Imaging data sets were co-registered according to anatomic match. Lymph nodes were contoured separately if they were not contiguous with the primary tumor. All volumes were delineated by one physician (PM), and $20 \%$ of them were randomly checked by a senior physician (FMK). The reproducibility of the system was completed by comparing volumes by the same physician using the same methodology within two systems (FIAT and UM PLAN) in the first ten consecutive cases, while the reproducibility of methodology was assessed by comparing volumes of these same patients between two physicians (PM and SY).

PET metabolic tumor volume delineation

There are multiple ways to define PET-MTVs. There are strengths and weaknesses of each methodology; tumor background method is considered to be one of the more reproducible methods. We elected to use an autosegmentation method based on a fixed source/background 
ratio, combined with $\mathrm{CT}$ anatomy-based manual editing to delineate PET-MTV as illustrated in Fig. 1. As the background blood pool is most commonly used as the reference for lung cancer diagnosis [16], we elected to use FDG uptake in the aorta to represent the normal activity of mediastinum background. To determine an optimal tumor/aorta ratio (TAR) value, we first completed a pilot study to measure the mean activity of $1 \mathrm{~cm}^{3}$ within the aortic arches on a pilot of 10 patients. Normalizing the mean aortic arch value to 1.0 , the upper limits of $95 \%$ and $99 \%$ confidence intervals for the aortic arch were 1.2 and 1.5, respectively. We then compared the PET-MTVs of various TARs ranging from 1.2 to 2.0 and found that PET-MTVs from TARs of 1.5 were most effective and reproducible as it associated with the least amount of manual editing for tumors adjacent to mediastinum or chest wall. The auto-segmented MTVs were inspected visually at every slice through the co-registered CT-PET images; areas of FDG uptake from normal structures such as artery, bone marrow, heart, and esophagus were manually edited out from the PET-MTVs (Fig. 2). The central necrosis holes of tumors were not included in the PET-MTVs because we were most interested in metabolic uptake volume which needs to be metabolically. The schema of MTV definition is shown in Fig. 1.

$\mathrm{CT}$ gross tumor volume delineation

The CT images without contrast from same PET-CT data sets were used for delineation. To improve the objectivity of the CT volume delineation, $\mathrm{CT}$ gross tumor volumes (CT-GTVs) were delineated using auto-segmentation (arbitrarily CT number $=500$ ). We then edited the CTGTVs by using anatomic guidance using mediastinum and lung windows, as appropriate. The spiculated branches of tumors were included, and central necrosis regions were filled (Fig. 3). Regions of suspected disease, such as hazy areas or controversial atelectasis areas, were also included in CT-GTVs.

Study objectives and data analysis

The primary endpoint of this study was MTVs on PET to GTVs on CT. Reproducibility of the methodology on the primary endpoint assessment, i.e., target delineation, is

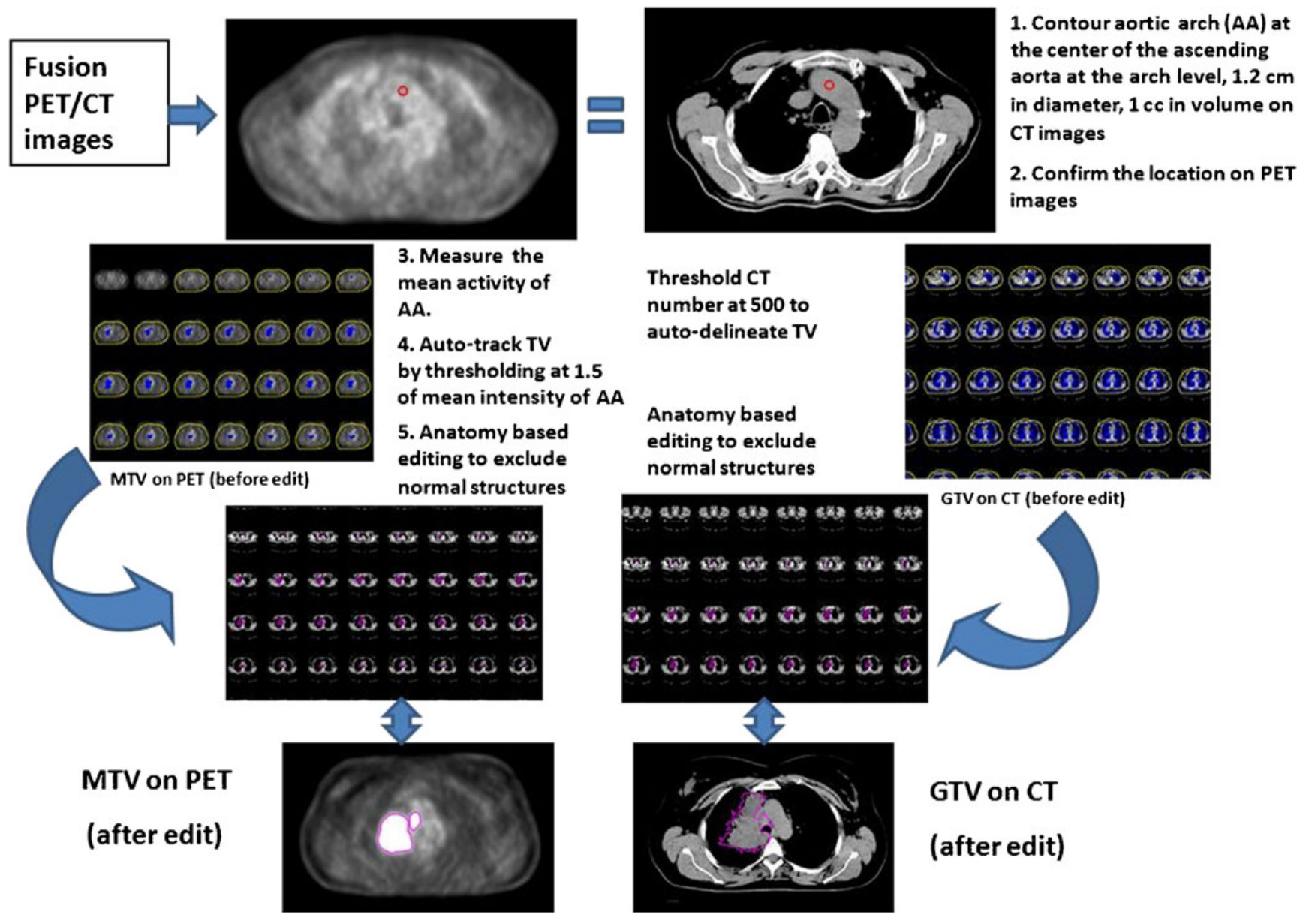

Fig. 1 Tumor delineation on PET/CT. $T V=$ tumor volume, $G T V=$ gross tumor volume, $M T V=$ metabolic tumor volume 
Fig. 2 TAR selection for PETMTV delineation. TAR $=$ tumor aorta ratio, $M T V=$ metabolic tumor volume. Example image shows PET-MTVs autosegmented by TAR 1.2 (light blue), 1.5 (dark blue), 1.7 (green), and 2.0 (red) on PET (a) and CT (b). TAR 1.5 was chosen as it appeared most appropriately and associated with the least amount of edit. PET-MTV auto-delineation by TAR 1.5 before edit (dark blue) and after edit (pink) by exclusion pulmonary aorta, esophagus, and bone marrow on PET image (c) and CT (d). $G T V=$ gross tumor volume, $M T V=$ metabolic tumor volume

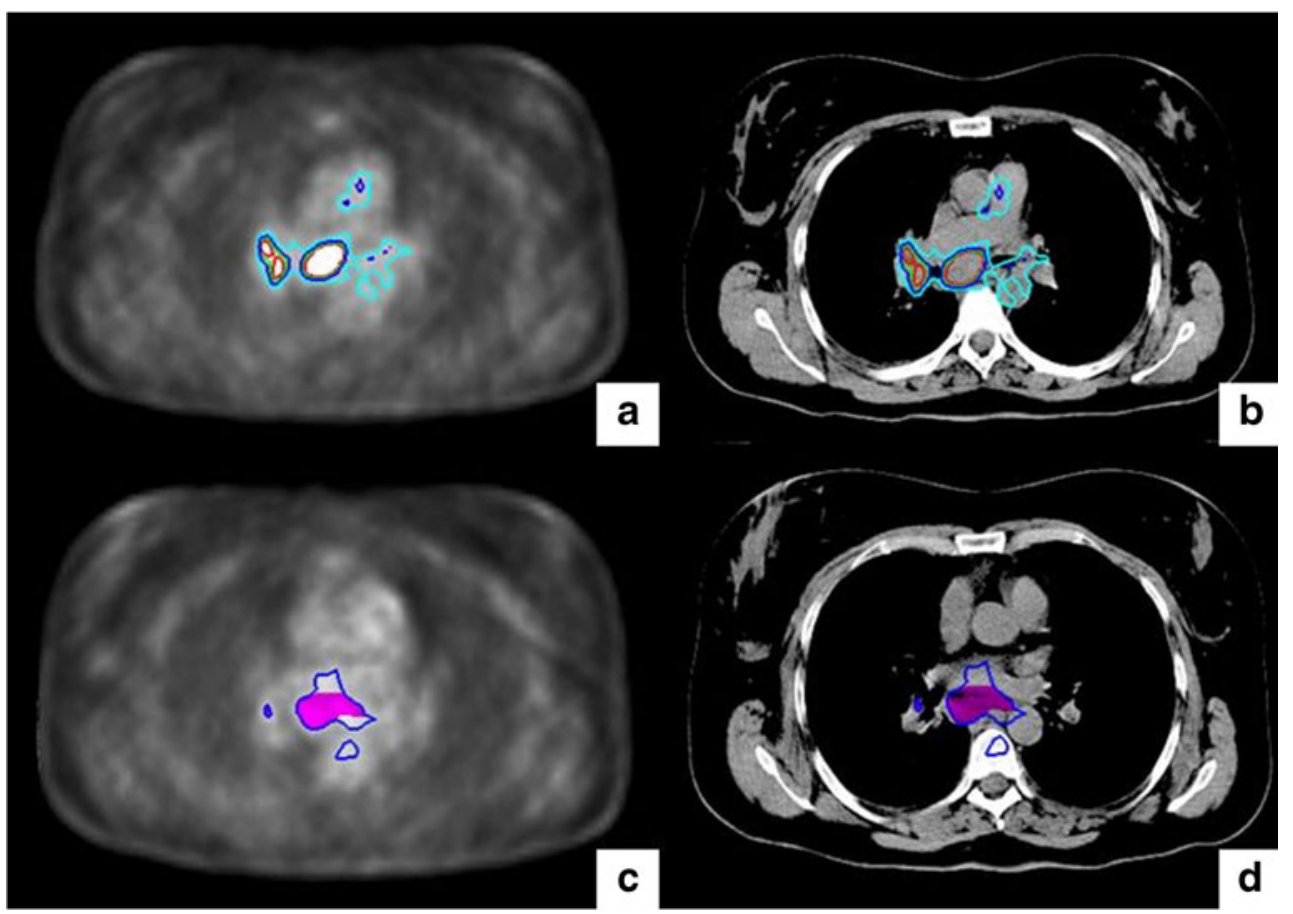

essential and was tested by linear correlation. The primary objective of this study was to compare the changes in PETMTVs and CT-GTVs between pre- and during-treatment, 3DCRT and SBRT. SPSS 13.0 software was used to test statistical significance. Intraclass coefficiency [17] was used to test the correlation between tumor volumes of two physicians from the same system and of one physician from two systems. The correlation between PET-MTVs and CT-GTVs
Fig. 3 Example tumor volumes. This figure shows a primary tumor with a central necrosis for MTV-PET (a) and CT-GTV (b). The MTV excludes the central necrosis (a) while the GTV includes the necrosis (b). GTV were autotracked on an axial CT image under a soft tissue window (c) and lung window (d). $G T V=$ gross tumor volume, $M T V=$ metabolic tumor volume

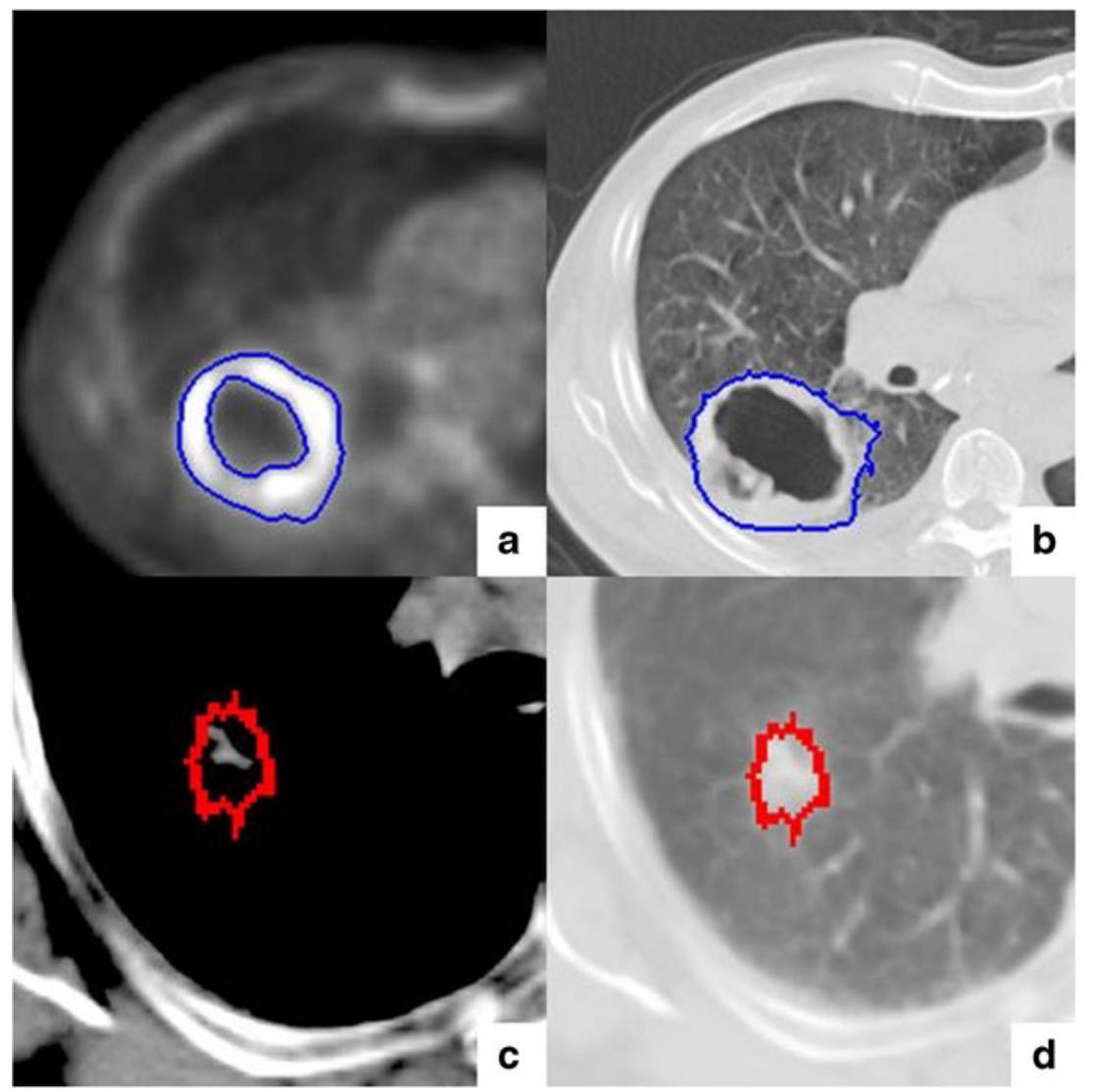


was tested using linear regression analysis; the change of each individual tumor during-RT was compared with that of pre-RT by two-tailed paired $t$ test. $P$ values equal to or less than 0.05 were considered to be statistically significant. Unless otherwise specified, the data are presented as mean $(95 \% \mathrm{CI})$.

\section{Results}

Fifty patients were enrolled in this study. There were a total of 88 lesions on CT and 86 on PET. The patient characteristics are shown in Table 1. Thirty-three patients $(66 \%)$ received concurrent chemo-radiotherapy, while the remaining patients received definitive radiation alone. Five patients (five lesions) with stage I disease were treated with SBRT. The median interval time between pre-RT and during-RT scan was 38 (range, 10-60) days, 12 (range, 10-31) days, and 41 (range, 26-60) days in all patients, SBRT patients, and 3DCRT patients, respectively.

Reproducibility of tumor definition methodology

Reproducibility was accomplished in the first ten consecutive patients in the system. PET-MTVs were delineated by one physician by using two systems (the FIAT and the UM

Table 1 Patient characteristics

\begin{tabular}{ll}
\hline Patients characteristics $(n=50)$ & \\
\hline Age (years) & \multicolumn{1}{c}{$(5-86$} \\
& $($ mean 67.9, median 68.1$)$ \\
Concurrent chemotherapy & $33(66 \%)$ \\
Yes & $17(34 \%)$ \\
No & \\
Gender & $37(74 \%)$ \\
Male & $13(26 \%)$ \\
Female & $9(18 \%)$ \\
Stage & $10(20 \%)$ \\
I & $31(62 \%)$ \\
II & $9(18 \%)$ \\
III & $8(16 \%)$ \\
Pathology & $1(2 \%)$ \\
Squamous cell carcinoma & $31(62 \%)$ \\
Adenocarcinoma & $1(2 \%)$ \\
Large cell & \\
NSCLC (not-specified) & $51^{\mathrm{a}}$ \\
No pathology & 35 \\
Tumor types (PET-based) & \\
Primary tumor &
\end{tabular}

${ }^{a}$ Three patients had two primaries. Two patients had no primary tumor systems) and by two physicians within the same system (FIAT) in the same ten patients for both pre- and duringRT images. Five patients had central lesions, and the remaining five had peripheral lesions. The intraclass correlation coefficiency (ICC) of PET-MTVs and CT-GTVs between two systems (one physician) was 0.98 (95\% CI, 0.96$0.99)$ and 0.98 (95\%CI, 0.96-0.99), respectively. The ICC was 0.99 (95\%CI, 0.99-0.99) and 0.98 (95\% CI, 0.97-0.99) between two physicians of the same system, for PET-MTVs and CT-GTVs, respectively (Fig. 4). PET-MTVs varied slightly more between the two systems than they did between two physicians (Fig. 4).

The change of tumor volumes on PET and CT during-RT

The mean ratios of MTV/GTV were $0.70(-0.07 \sim 1.47)$ and $0.33(-0.30 \sim 0.95)$ for pre-RT and during-RT, respectively. Table 2 shows tumor volumes (cubic centimeters and percentage) on PET/CT images obtained pre-RT and during-RT as well as differences CT-PET volumes between the studies. The mean CT-GTVs were $84.1 \mathrm{cc}(54.2-114.0 \mathrm{cc})$ and $50.1 \mathrm{cc}(34.2-66.0 \mathrm{cc})$, while the mean PET-MTVs were $43.4 \mathrm{cc}(28.2-58.5 \mathrm{cc})$ and $17.9 \mathrm{cc}(10.0-25.7 \mathrm{cc})$ on pre-RT and during-RT scan, respectively. The tumor volume reduced significantly during-RT on both PET and CT images. The mean reductions of PET-MTVs and CT-GTVs were $32.2 \mathrm{cc}(20.8-43.7 \mathrm{cc})$ and $40.7 \mathrm{cc}(18.8-62.7 \mathrm{cc})$ (paired $t$ test, $p<0.001)$, respectively. PET-MTVs had a significantly greater proportional reduction (mean, $70 \%$; $95 \%$ CI $62-$ $77 \%$ ) than CT-GTVs (mean, $41 \%$; 95\% CI 33-49 \%, $p<$ 0.001) (Fig. 5). Interestingly, $3 / 85$ lesions had their PETMTV enlarged during RT. Two of them were lower lung lesions treated with SBRT (from $28.4 \mathrm{cc}$ to $35.7 \mathrm{cc}$ in one, $3.3 \mathrm{cc}$ to $5.3 \mathrm{cc}$ in another). The other lesion was a subcarinal node after 3DCRT, from $2.9 \mathrm{cc}$ from pre-RT to 4.10 cc during-RT.

Factors associated with tumor volume reduction during-RT

There were remarkable individual heterogeneities in the magnitude of changes in tumor volumes on both $\mathrm{CT}$ and PET (Fig. 5). Compared with that of primary tumors (Fig. 6), lymph nodes appeared to have a significantly greater percentage reduction in both PET-MTVs and CT-GTVs though, overall, there was a significant correlation between changes in PET-MTVs and changes in CT-GTVs with a Pearson's correlation coefficient of 0.55 ( $p<0.001$ Fig. 6). The mean PET-MTV reduction was $61.4 \%(52.2-70.5 \%)$ and 81.4 $(70.9-91.9 \%)$ for primary tumors and lymph nodes $(p=$ $0.007)$, respectively. The mean CT-GTV reduction was $31.3 \%(20.1-42.6 \%)$ and $54.0 \%(43.3-64.6 \%)$ for primary tumors and lymph nodes $(p=0.007)$, respectively. Other factors were also evaluated for their association with the changes 
Fig. 4 Reproducibility of tumor delineation methodology a MTV in the UM planning system (red line); $\mathbf{b}$ MTV in the FIAT image analysis system (red body) for the same patient; c MTVs drawn by two physicians in the same system (red body and black line) in a different patient with low central tumor activity; $\mathbf{d}$ GTVs drawn by two physicians (red and blue lines); e correlation of MTV and GTV between the two systems by a same physician; $\mathbf{f}$ correlation of MTV and GTV between the two physicians. $G T V=$ gross tumor volume, $M T V=$ metabolic tumor volume. $G T V=$ gross tumor volume, $M T V=$ metabolic tumor volume, $I C C=$ correlation coefficiency
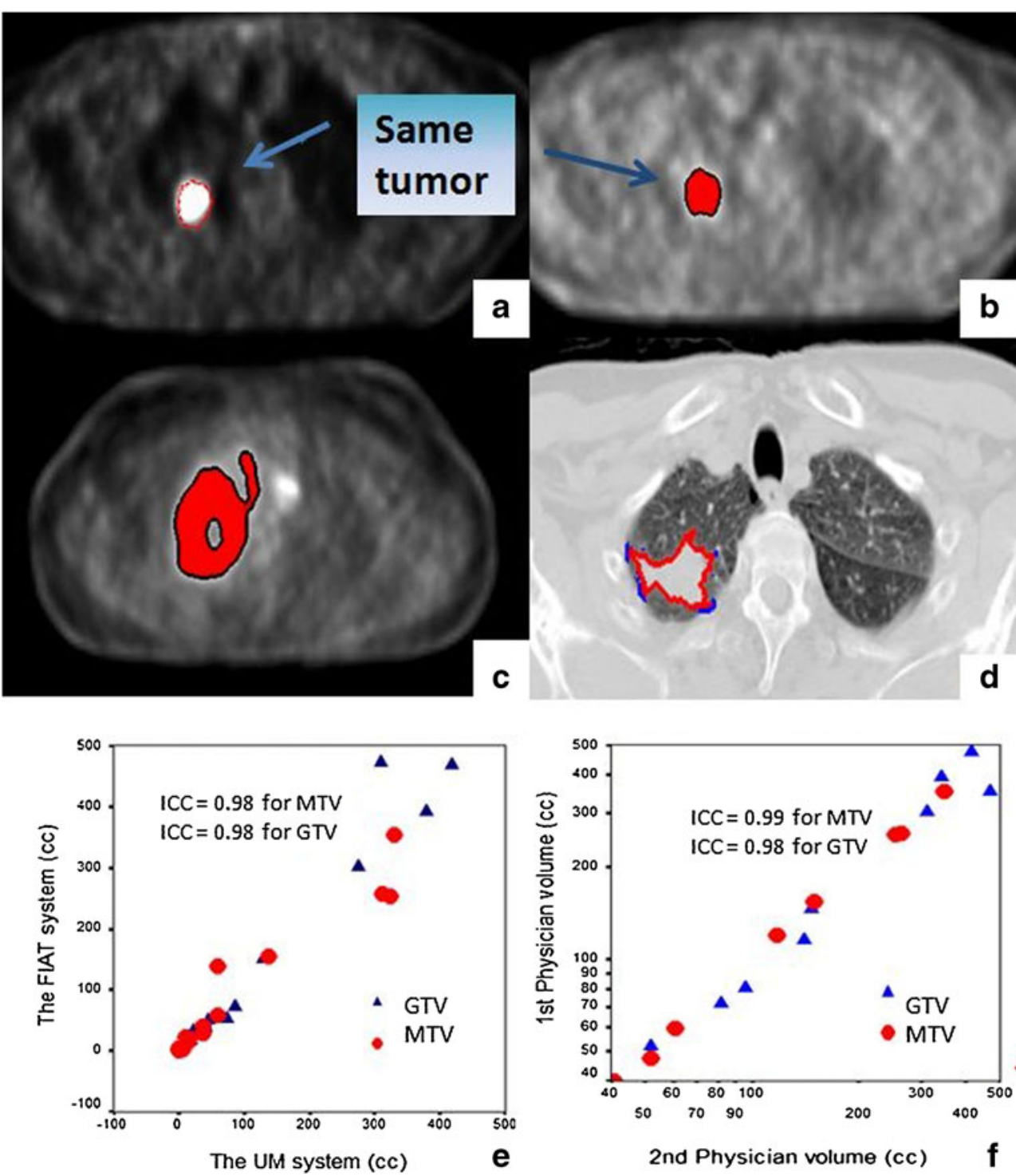

of PET-MTVs during-RT (Table 3). There was no significant correlation between changes (percent) of PET-MTVs and estimated diameter of pre-RT PET-MTVs and CT-GTVs.
Estimated diameter of volume was calculated by $4 / 3 \rrbracket R^{3}$ equation $(R=$ diameter, $\boldsymbol{q}=22 / 7)$. The percentage change in PETMTVs was also significantly correlated with type of RT

Table 2 MTV and GTV pre-RT and during-RT in all patients

\begin{tabular}{|c|c|c|c|c|c|c|c|c|c|}
\hline \multirow[t]{2}{*}{ Value } & \multicolumn{3}{|c|}{ Pre-RT } & \multicolumn{3}{|c|}{ During RT } & \multicolumn{3}{|c|}{ Volume difference } \\
\hline & Mean & Range & $95 \% \mathrm{CI}$ of mean & Mean & Range & $\begin{array}{l}95 \% \mathrm{CI} \\
\text { of mean }\end{array}$ & Mean & $\begin{array}{l}95 \% \mathrm{CI} \text { of the } \\
\text { difference }\end{array}$ & $\begin{array}{l}p \\
\text { value }\end{array}$ \\
\hline PET-MTV (cc) & 50.1 & $0.1 \sim 352.4$ & $34.2 \sim 66.0$ & 17.9 & $0 \sim 256.3$ & $10.0 \sim 25.7$ & 32.2 & $20.8 \sim 43.7$ & $<0.001$ \\
\hline $\begin{array}{l}\text { Percentage of } \\
\text { PET-MTV to pre-RT }\end{array}$ & 100 & $100 \sim 100$ & $100 \sim 100$ & 30.5 & $0 \sim 158.1$ & $23.3 \sim 37.7$ & 69.5 & $62.2 \sim 76.8$ & $<0.001$ \\
\hline CT-GTV (cc) & 84.1 & $0.5 \sim 815.6$ & $54.2 \sim 114.0$ & 43.4 & $0 \sim 471.73$ & $28.2 \sim 58.5$ & 40.7 & $18.8 \sim 62.7$ & $<0.001$ \\
\hline $\begin{array}{l}\text { Percentage of } \\
\text { CT-GTV to pre-RT }\end{array}$ & 100 & $100 \sim 100$ & $100 \sim 100$ & 59.2 & $0 \sim 193.2$ & $51.0 \sim 67.4$ & 40.8 & $32.5 \sim 49.2$ & $<0.001$ \\
\hline $\begin{array}{l}\text { Difference of } \\
\text { GTVs-MTVs (cc) }\end{array}$ & 35.8 & $-53.7 \sim 772.0$ & $\begin{array}{r}14.1 \sim 57.6 \\
p=0.002\end{array}$ & 26.4 & $-0.64 \sim 260.3$ & $\begin{array}{c}16.2 \sim 36.6 \\
p<.001\end{array}$ & 9.43 & $-9.5 \sim 28.4$ & 0.325 \\
\hline $\begin{array}{l}\text { Difference of GTV } \\
\text { and MTV }(\%)\end{array}$ & 29.9 & $-84.1 \sim 96.4$ & $21.6 \sim 38.2$ & 65.1 & $-13.1 \sim 100$ & $57.9 \sim 72.3$ & 34.6 & $25.1 \sim 44.1$ & $<0.001$ \\
\hline
\end{tabular}


Fig. 5 Changes of PET-MTV and CT-GTV on PET/CT imaging during-RT. $G T V=$ gross tumor volume, $M T V=$ metabolic tumor volume. a Shows the mean MTV and GTV pre- and during-RT; $\mathbf{b}$ shows the absolute difference of MTV and GTV between pre- and duringRT; $\mathbf{c}$ shows percentage changes of each individual PET-MTV and CT-GTV. GTV= gross tumor volume, $M T V=$ metabolic tumor volume a

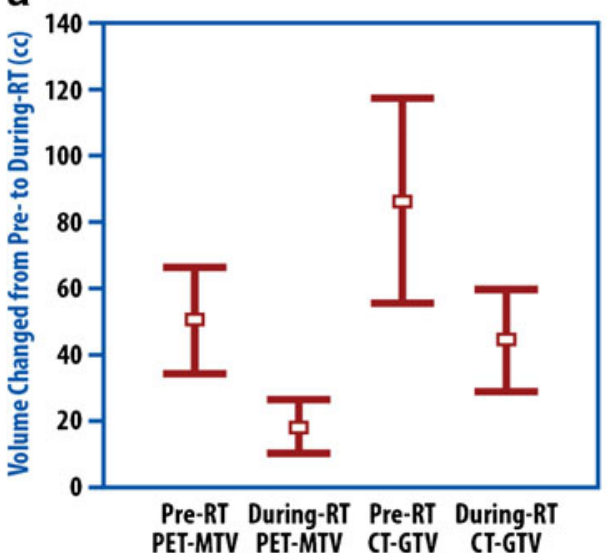

Pre-RT During-RT Pre-RT During-RT
PET-MTV PET-MTV CT-GTV CT-GTV

C

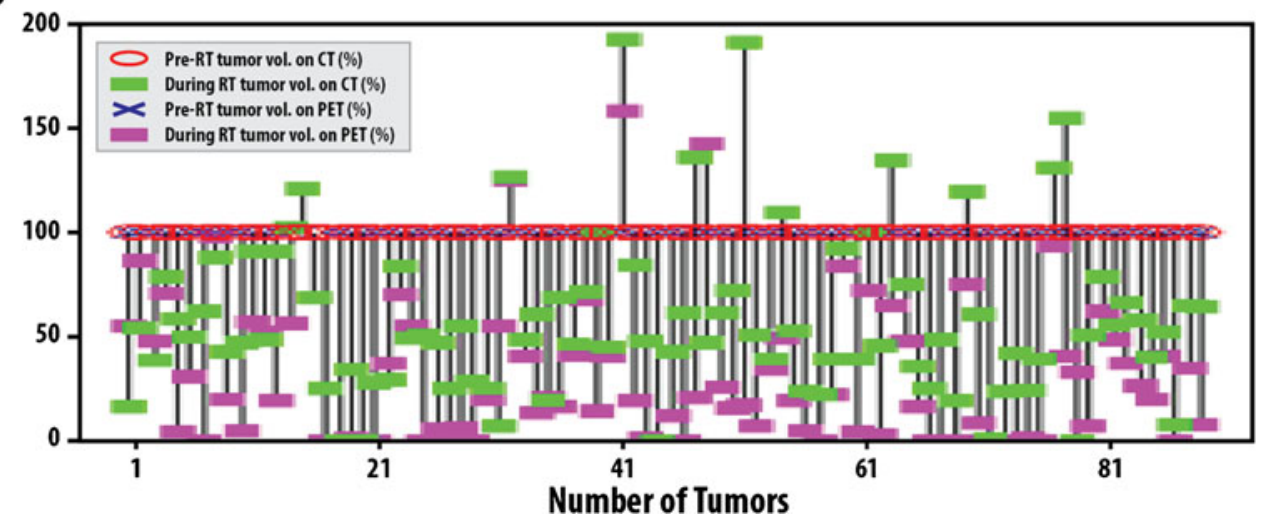

(conventional fractionation versus SBRT, $r^{2}=0.40, p<0.001$ ), concurrent chemotherapy $\left(r^{2}=0.24, p=0.029\right)$, maximum FDG activity of tumor at baseline $\left(r^{2}=0.24, p=0.002\right)$, maximum normalized tumor activity $(\mathrm{NTA}=$ tumor activity divided by the mean aorta activity) $\left(r^{2}=0.28, p=0.009\right)$ and mean NTA $\left(r^{2}=\right.$ $0.25, p=0.02$ ) (Table 3 ). Patients who received conventional treatment had a significantly greater reduction (mean $72.9 \%$, $95 \%$ CI of mean 66.4-79.4\%) in PET-MTVs than patients who were treated with SBRT (mean, $15.4 \%$, $95 \%$ CI of mean $-31.6-62.5 \%)(p<0.001)$ (Table 4$)$. There was a significantly greater reduction in PET-MTVs in patients who had mean NTA $\leq 2.5$ (mean 79.2 \%, $95 \%$ CI of mean $69.2-89.2 \%$ ) than patients who had mean NTA $>2.5$ (mean $61.5 \%, 95 \%$ CI of mean 51.8-71.2\%) $(p=0.015)$. Multivariate analysis showed that low maximum NTA $(p=0.026)$ and type of treatment (conventional RT versus SBRT) $(p<0.001)$ were significantly correlated with greater changes (percent) in PET-MTVs.

\section{Discussion}

In this prospective study, we demonstrated a reproducible method of tumor target delineation by combining auto-threshold and manual editing on PET. Using this methodology, we have further demonstrated that MTVs on PET and GTVs on CT reduce

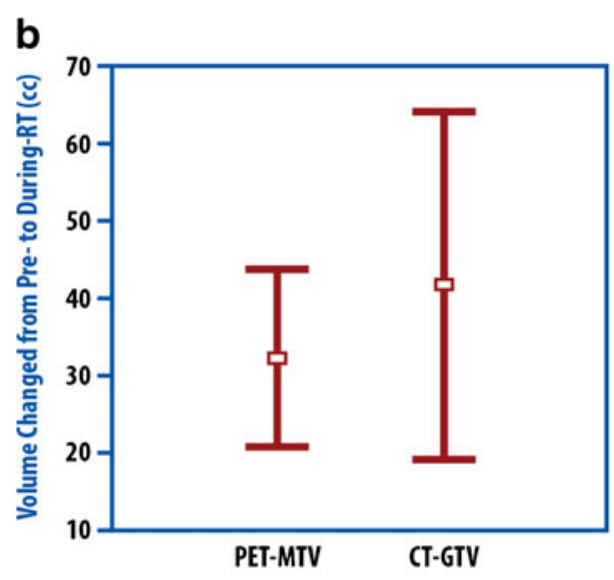

significantly during the course of RT. While there was a remarkable heterogeneity in magnitude of volume reduction, there was a significant correlation between reductions of PET-MTV and CT-GTV. Patients with less active tumors and treated with chemotherapy were associated with a greater volume reduction during-RT, and 3DCRT had a greater reduction during-RT than SBRT.

PET-MTV delineation is challenging, and there is no universally acceptable method. Some authors use a percentage of the maximum or peak activity, whereas others recommend an absolute SUV value (e.g., an SUV threshold of 2.5 [18] to represent the edge of the lesion). It is now known that a fixed threshold method of using 40-50\% of maximum activity may lead to significant errors in the volume estimation [19]. A volumetric comparison of four methods (visual, $40 \%$ maximum activity, SUV2.5, and source/background ratio $(\mathrm{S} / \mathrm{B}))$ in primary NSCLC showed substantially different volumes from different techniques and application of $\mathrm{S} / \mathrm{B}$ ratios generated the most reasonable volumes, comparable to breath-expanded CT volumes [1]. Van Baardwijk [20] and colleagues attempted to compare S/B-based PET$\mathrm{CT}$ auto-delineation. They reported a good correlation with pathology (correlation coefficient $=0.90)$, decreased the delineated volumes of the GTVs, and reduced the interobserver variability. Auto-contoured GTVs were smaller than manually 
Fig. 6 Factors associated with PET-MTV changes during-RT. PET-MTV reduction is significantly correlated with the reduction in CT-GTV (a). Lymph nodes have a significantly greater reduction in both PET-MTVs and CTGTVs during-RT (b). Examples scans include: Metabolic complete response (CR) in lymph node MTV (c and d). Metabolic complete response in primary tumor after concurrent chemo-RT (e and f). Metabolic stable response in SBRT case ( $\mathbf{g}$ and $\mathbf{h}) . G T V=$ gross tumor volume, $M T V=$ metabolic tumor volume, $L N=$ lymph node
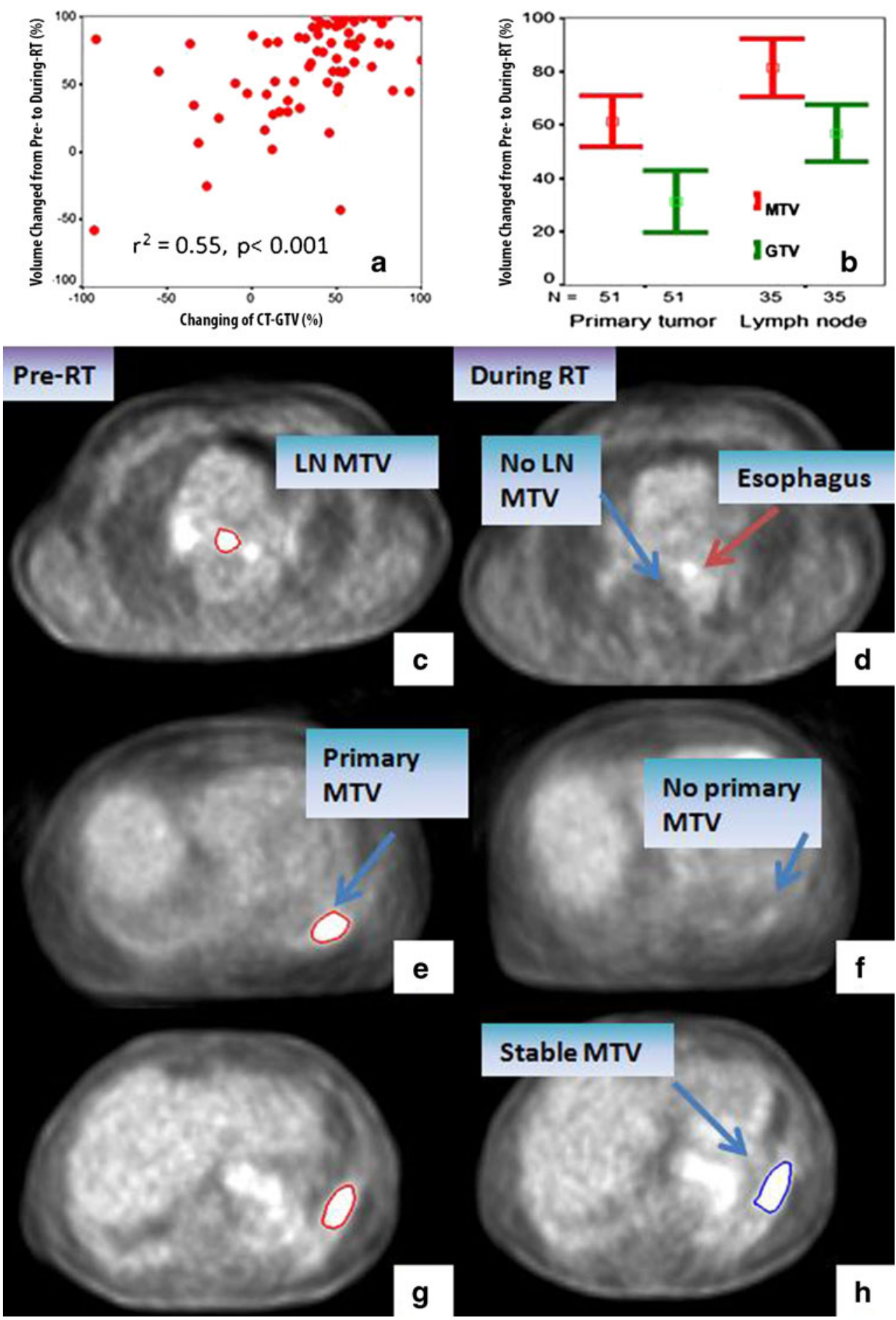

contoured ones. In another study, the same group found that tumors auto-contoured at $42 \%$ of maximum level overestimated the PET tumor volume in two of five cases, while CTGTVs were larger than pathologic volume in four of five cases [21]. A pilot study comparing tumor volumes as determined by pathologic examination and FDG-PET/CT images of NSCLC showed that the optimal threshold and absolute SUV were $31 \% \pm 11 \%$ and $3.0 \pm 1.6$, respectively [22]. Furthermore, several other studies showed that with PET-defined tumor volumes varied significantly with the methodology, resulting in considerable inter-observer and intra-observer variations [23-25]. Fused PET and CT altered volume in about $50 \%$ patients compared with $\mathrm{CT}$ volume alone, either by visual evaluation or using some mathematical algorithm, such as a fixed standard uptake value or threshold [24, 26, 27]. The relationship between PET-based (15\% or $40 \%$ of the maximal 
Table 3 Factors associated with PET-MTVs reduction during-RT

\begin{tabular}{lll}
\hline Factors & $r^{2}$ & $p$ values \\
\hline Stage & 0.16 & 0.13 \\
RT (conventional or SBRT) & 0.40 & $<0.001$ \\
Pre-RT PET-MTV & 0.10 & 0.35 \\
Pre-RT CT-GTV & 0.10 & 0.36 \\
Pre-RT MTV diameter & 0.17 & 0.11 \\
Pre-RT GTV diameter & 0.18 & 0.10 \\
Chemotherapy (yes or no) & 0.24 & 0.029 \\
Tumor maximum activity & 0.24 & 0.002 \\
Tumor mean activity & 0.15 & 0.17 \\
Maximum NTA & 0.28 & 0.009 \\
Mean NTA & 0.25 & 0.02 \\
\hline
\end{tabular}

NS not significant, MTV metabolic tumor volume, GTV gross tumor volume, NTA normalized tumor activity

iso-uptake value threshold methods) and CT-based volumes (visual method) generally suffers from poor correlation between the two image data sets, expressed in terms of a large statistical variation in gross tumor volume ratios, irrespective of the threshold method used [28]. With pathologic examination, the contour of the tumor volume of NSCLC patients with co-registered FDG-PET/CT resulted in $>50 \%$ alterations compared with CT targeting [29]. Recently, Bayne et al. demonstrated that PET-MTV autocontours generated using SUV 2.5, 3.5, and $40 \%$ SUVmax differed widely in each of six cases and recommended a visual contouring protocol for contouring MTV in NSCLC [30] whereas comparative assessment in an anthropomorphic phantom demonstrated that method by background activity and a model-based method were more accurate and reproducible than $\mathrm{SUV}_{\max }$ [31]. There has not been a study using the same method to test the reproducibility of different software systems or between different physicians. We elected the tumor/background ratio methodology, since it could be the most reasonable automated method [1, 20]. Although some physicians believe that normal liver standardized uptake value normalized for lean body mass (SUL) is slightly more stable than determinations of blood-pool SUL [32], FDG uptake at aorta was selected to be representative of background in this study because increased uptake area greater than mediastinal blood pool was often defined as abnormal findings [16] or residual tumor [33]. We found that the mean uptake at the aorta was reproducible even if the center of ROI in each slide was slightly shifted to within the wall of an aorta (in the pilot study). Both central $(50 \%)$ and peripheral $(50 \%)$ lesions were studied. There has not been a study using the same method to test the reproducibility of different software systems and between different physicians. Our methodology of delineating tumor volumes on

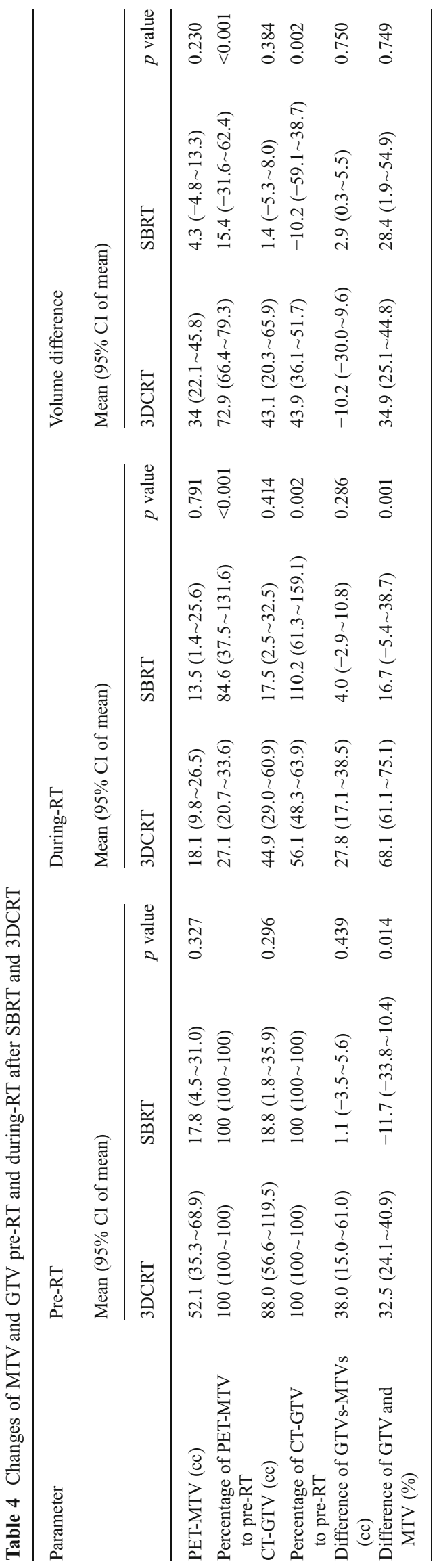


PET and CT generated a very high ICC value, which suggests that this method of combining complex TAR auto-contouring method and manual editing may be superior to methods using a simple cutoff (SUV or percent threshold).

The current study is among the first to extensively examine PET-MTVs in comparison to CT-GTVs during-RT. This study demonstrates a significant tumor volume reduction during-RT on both PET and CT. On average, PET-MTVs changed significantly more than CT-GTVs $(p<0.001)$. The mechanism behind such differences is unclear, while underlying biology of each tumor could be part of the etiology. That MTVs from functional imaging (PET scans) changed more than GTVs on CT scan may suggest that tumor functional activity changes earlier or faster than morphologic appearance on CT. Indeed, the vast majority of tumors had greater reduction on PET-MTVs than CT-GTVduring-RT, despite the fact that PET-MTVs may have also included motion. This is important as it further suggests the value of using PET-MTV during-RT for dose escalation in supplement to CT-GTV-based adaptive RT or using dose painting on biologic planning target volume [34, 35]. RTOG1106 has been activated to adapt radiation therapy based on during-RT PET-MTV.

Patients treated with SBRT also had reduced PET-MTV and CT-GTV during-RT. This is remarkable as the PET scan during-RT for this group of patients could be performed as early as $3-5$ days from SBRT start. It is also interesting to note that SBRT had significantly less reduction in PET-MTV than those received conventional fractionated RT. The mechanism of this is unclear. It could be a result of not enough time to allow tumor response as the during-SBRT PET was normally performed at 1-2 weeks (median 12 days) from SBRT start. Or it could be due to a slower effect of SBRT on PET-CT in NSCLC. Respiratory motion and size may have also impacted tumor quantification and delineation in PET/CT imaging [36], which may partially explain that two small lesions of SBRT cases in lower lung lobe increased in tumor volume. Vahdat et al. [37] studied FDG-PET/CT serial tumor response in 20 stage IA NSCLC patients and demonstrated that tumor $\mathrm{SUV}_{\max }$ values return to background levels at 18-24 months following treatment. CT tumor shrinkage also continued for $2-15$ months after SBRT [38]. No SBRT study has previously reported on MTV reduction during-RT. On the other hand, modest reduction of MTV during-SBRT versus excellent tumor control after SBRT may suggest that PET scan during-SBRT may not be a good predictor for long-term outcome. On other side, it may deserve further study whether such a volume difference can convert an otherwise unsafe plan to a safe one for normal tissue tolerance. A study with a larger number of SBRT cases is needed.

It is worth mentioning that lymph nodes had greater volumetric changes on both PET and CT as compared with primary tumors, after the same dose of radiation. Initially, we thought this was a result of volume effect, as the primary tumors are larger; thus the same amount of absolute volume reduction would cause less change in percentage. However, we failed to detect a correlation between tumor volume reduction during-RT and tumor volume at baseline. Further study is needed to validate this finding and investigate the underlying mechanism of this phenomenon.

There are also remarkable individual differences in changes of tumor volume during-RT. Those receiving concurrent chemotherapy, lower maximum tumor FDG activity, lower maximum NTA, and lower mean NTA were significantly correlated with higher percentage of PET tumor volume changing. It is possible that heterogeneity in the nature of tumors responding to treatment is due to biology or genetic heterogeneity and may be further associated with the prognosis. A Japanese study demonstrated that SUVs on both early and delayed scans (early scanning at $1 \mathrm{~h}$ and delayed scanning at $2 \mathrm{~h}$ ) after treatment were significantly lower in pathologic responders than in non-responders $(p=0.0005$ and $p=0.0015$, respectively) [39]. Pottgen et al. also found a significantly greater percentage reduction in the SUVmax in patients showing an excellent pathologic response in the primary tumor than in those with greater than $10 \%$ residual viable cells $(p<0.005)$ after receiving neoadjuvant chemotherapy or chemoradiotherapy [40].

There were limitations to this study. The CT images from $\mathrm{PET} / \mathrm{CT}$ fusion images were performed without intravenous (IV) contrast media, which may decrease the accuracy of the CT-GTV delineations. In practice, CT-simulation with IV contrast and using RT treatment position co-registered with planning CTs [41] could improve contouring.

\section{Conclusion}

In summary, we have demonstrated a reproducible method to delineate tumor on PET/CT images. From a study of 50 patients, we demonstrated that metabolic tumor volumes on PET reduced more than GTVs on CT during-RT, suggesting that functional volumes reduce more rapidly than physiological volume. Using PET-volumes during-RT to escalate the radiation dose or calculate for dose painting radiotherapy in patients with non-small lung cancer could be of value in the future. Prospective clinical trials such as RTOG1106 and UMCC 2007123 are ongoing to individualize adaptive RT dose escalation in each patient based on these methods and results.

Acknowledgment This study was partially supported by American Society of Clinical Oncology (ASCO) Career Developmental Award, NIH grants R21CA127057 and P01CA59827. 
Conflict of interest None of the authors have any or potential conflict of interest in connection with this paper.

\section{References}

1. Nestle U, Kremp S, Schaefer-Schuler A et al (2005) Comparison of different methods for delineation of 18 F-FDG PET-positive tissue for target volume definition in radiotherapy of patients with non-small cell lung cancer. J Nucl Med 46:1342-1348

2. Yu HM, Liu YF, Hou M et al (2009) Evaluation of gross tumor size using CT, 18 F-FDG PET, integrated 18 F-FDG PET/CT and pathological analysis in non-small cell lung cancer. Eur J Radiol 72:104-113

3. Macmanus M, Nestle U, Rosenzweig KE et al (2008) Use of PET and PET/CT for radiation therapy planning: IAEA expert report 2006-2007. Radiother Oncol 87:142-146

4. Gong HY, Yu JM, Fu Z (2006) [Impact of PET/CT on precise radiotherapy planning for non-small cell lung cancer]. Zhonghua Zhong Liu Za Zhi 28:54-57

5. Lavrenkov K, Partridge M, Cook G et al (2005) Positron emission tomography for target volume definition in the treatment of nonsmall cell lung cancer. Radiother Oncol 77:1-4

6. Bradley JD, Perez CA, Dehdashti F et al (2004) Implementing biologic target volumes in radiation treatment planning for nonsmall cell lung cancer. J Nucl Med 45(Suppl 1):96S-101S

7. Nestle U, Kremp S, Grosu AL (2006) Practical integration of [18 F]FDG-PET and PET-CT in the planning of radiotherapy for non-small cell lung cancer (NSCLC): the technical basis, ICRU-target volumes, problems, perspectives. Radiother Oncol 81:209-225

8. Mac Manus MP, Hicks RJ (2007) Impact of PET on radiation therapy planning in lung cancer. Radiol Clin North Am 45:627$638, \mathrm{v}$

9. Nestle U, Walter K, Schmidt S et al (1999) 18 F-Deoxyglucose positron emission tomography (FDG-PET) for the planning of radiotherapy in lung cancer: high impact in patients with atelectasis. Int J Radiat Oncol Biol Phys 44:593-597

10. Kong FM, Frey KA, Quint LE et al (2007) A pilot study of [18 F] fluorodeoxyglucose positron emission tomography scans during and after radiation-based therapy in patients with non small-cell lung cancer. J Clin Oncol 25:3116-3123

11. Feng M, Kong FM, Gross M et al (2009) Using fluorodeoxyglucose positron emission tomography to assess tumor volume during radiotherapy for non-small-cell lung cancer and its potential impact on adaptive dose escalation and normal tissue sparing. Int J Radiat Oncol Biol Phys 73:1228-1234

12. Aristei C, Falcinelli L, Palumbo B et al (2010) PET and PET-CT in radiation treatment planning for lung cancer. Expert Rev Anticancer Ther 10:571-584

13. Mac Manus M, Hicks RJ (2008) The use of positron emission tomography (PET) in the staging/evaluation, treatment, and follow-up of patients with lung cancer: a critical review. Int J Radiat Oncol Biol Phys 72:1298-1306

14. Fox J, Ford E, Redmond K et al (2009) Quantification of tumor volume changes during radiotherapy for non-small-cell lung cancer. Int J Radiat Oncol Biol Phys 74:341-348

15. Spoelstra FO, Pantarotto JR, van Sornsen de Koste JR et al (2009) Role of adaptive radiotherapy during concomitant chemoradiotherapy for lung cancer: analysis of data from a prospective clinical trial. Int J Radiat Oncol Biol Phys 75:1092-1097

16. Asad S, Aquino SL, Piyavisetpat N et al (2004) False-positive FDG positron emission tomography uptake in nonmalignant chest abnormalities. AJR Am J Roentgenol 182:983-989
17. Fleiss JL (ed) (1981) Statistical methods for rates and proportions, 2nd edn. Wiley, New York

18. Hong R, Halama J, Bova D et al (2007) Correlation of PET standard uptake value and CT window-level thresholds for target delineation in CT-based radiation treatment planning. Int J Radiat Oncol Biol Phys 67:720-726

19. Yaremko B, Riauka T, Robinson D et al (2005) Threshold modification for tumour imaging in non-small-cell lung cancer using positron emission tomography. Nucl Med Commun 26:433-440

20. van Baardwijk A, Bosmans G, Boersma L et al (2007) PET-CTbased auto-contouring in non-small-cell lung cancer correlates with pathology and reduces interobserver variability in the delineation of the primary tumor and involved nodal volumes. Int $\mathrm{J}$ Radiat Oncol Biol Phys 68:771-778

21. Stroom J, Blaauwgeers H, van Baardwijk A et al (2007) Feasibility of pathology-correlated lung imaging for accurate target definition of lung tumors. Int J Radiat Oncol Biol Phys 69:267-275

22. Yu J, Li X, Xing L et al (2009) Comparison of tumor volumes as determined by pathologic examination and FDG-PET/CT images of non-small-cell lung cancer: a pilot study. Int J Radiat Oncol Biol Phys 75:1468-1474

23. Caldwell CB, Mah K, Ung YC et al (2001) Observer variation in contouring gross tumor volume in patients with poorly defined non-small-cell lung tumors on CT: the impact of 18FDG-hybrid PET fusion. Int J Radiat Oncol Biol Phys 51:923-931

24. Giraud P, Elles S, Helfre S et al (2002) Conformal radiotherapy for lung cancer: different delineation of the gross tumor volume (GTV) by radiologists and radiation oncologists. Radiother Oncol 62:27-36

25. Van de Steene J, Linthout N, de Mey J et al (2002) Definition of gross tumor volume in lung cancer: inter-observer variability. Radiother Oncol 62:37-49

26. Bradley J, Thorstad WL, Mutic S et al (2004) Impact of FDG-PET on radiation therapy volume delineation in non-small-cell lung cancer. Int J Radiat Oncol Biol Phys 59:78-86

27. Daisne JF, Sibomana M, Bol A et al (2003) Tri-dimensional automatic segmentation of PET volumes based on measured source-to-background ratios: influence of reconstruction algorithms. Radiother Oncol 69:247-250

28. Devic S, Tomic N, Faria S et al (2010) Defining radiotherapy target volumes using (18)F-fluoro-deoxy-glucose positron emission tomography/computed tomography: still a Pandora's box? Int J Radiat Oncol Biol Phys 78:1555-1562

29. Faria SL, Menard S, Devic S et al (2008) Impact of FDG-PET/CT on radiotherapy volume delineation in non-small-cell lung cancer and correlation of imaging stage with pathologic findings. Int $\mathrm{J}$ Radiat Oncol Biol Phys 70:1035-1038

30. Bayne M, Hicks RJ, Everitt S et al (2010) Reproducibility of "intelligent" contouring of gross tumor volume in non-small-cell lung cancer on PET/CT images using a standardized visual method. Int J Radiat Oncol Biol Phys 77:1151-1157

31. Tylski P, Stute S, Grotus N et al (2010) Comparative assessment of methods for estimating tumor volume and standardized uptake value in (18)F-FDG PET. J Nucl Med 51:268-276

32. Wahl RL, Jacene H, Kasamon Y et al (2009) From RECIST to PERCIST: evolving considerations for PET response criteria in solid tumors. J Nucl Med 50(Suppl 1):122S-150S

33. Mac Manus MP, Hicks RJ, Matthews JP et al (2003) Positron emission tomography is superior to computed tomography scanning for response-assessment after radical radiotherapy or chemoradiotherapy in patients with non-small-cell lung cancer. J Clin Oncol 21:1285-1292

34. Devic S, Tomic N, Faria S et al (2007) Impact of 18FDG-PET/CT on biological target volume (BTV) definition for treatment planning for non-small cell lung cancer patients. Nuclear Inst Methods Phys Res A 571:89-92 
35. Tanderup K, Olsen DR, Grau C (2006) Dose painting: art or science? Radiother Oncol 79:245-248

36. Liu C, Pierce LA 2nd, Alessio AM et al (2009) The impact of respiratory motion on tumor quantification and delineation in static PET/CT imaging. Phys Med Biol 54:7345-7362

37. Vahdat S, Oermann EK, Collins SP et al (2010) CyberKnife radiosurgery for inoperable stage IA non-small cell lung cancer: 18 F-fluorodeoxyglucose positron emission tomography/ computed tomography serial tumor response assessment. J Hematol Oncol 3:6

38. Aoki T, Nagata Y, Negoro Y et al (2004) Evaluation of lung injury after three-dimensional conformal stereotactic radiation therapy for solitary lung tumors: CT appearance. Radiology 230:101-108
39. Yamamoto Y, Nishiyama Y, Monden T et al (2006) Correlation of FDG-PET findings with histopathology in the assessment of response to induction chemoradiotherapy in non-small cell lung cancer. Eur J Nucl Med Mol Imaging 33:140-147

40. Pottgen C, Levegrun S, Theegarten D et al (2006) Value of 18 Ffluoro-2-deoxy-D-glucose-positron emission tomography/computed tomography in non-small-cell lung cancer for prediction of pathologic response and times to relapse after neoadjuvant chemoradiotherapy. Clin Cancer Res 12:97-106

41. Grgic A, Nestle U, Schaefer-Schuler A et al (2009) FDG-PETbased radiotherapy planning in lung cancer: optimum breathing protocol and patient positioning - an intraindividual comparison. Int J Radiat Oncol Biol Phys 73:103-111 